\title{
Drug-Related Polyarthralgias with Levodopa-Carbidopa
}

\author{
Shin C. Beh, MD*
}

Department of Neurology, University of Texas Southwestern Medical Center, Dallas, TX, USA

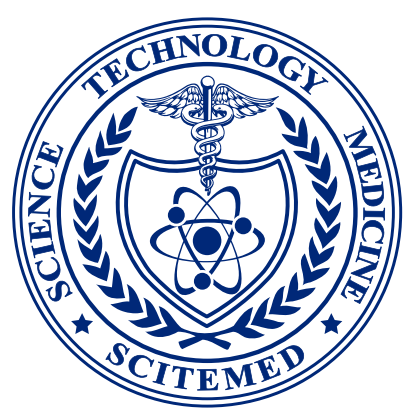

\section{ABSTRACT}

This case report characterizes the clinical course of a 79-year-old man with idiopathic Parkinson disease who developed polyarthralgia following commencement of levodopa-carbidopa therapy, resolution of the polyarthralgia with discontinuation of it, and subsequent reappearance of polyarthralgia when the medication was restarted. The time-locked relationship between polyarthralgia and levodopa-carbidopa treatment suggests that this is an adverse drug reaction. The possible pathophysiologic basis of this unusual reaction is discussed.
\end{abstract}

\section{INTRODUCTION}

Levodopa-carbidopa is the mainstay of treatment of Parkinson disease (PD). While there are reports of arthralgias arising from levodopa-carbidopa therapy, to my knowledge, this is the first detailed description of a time-locked occurrence of polyarthralgias with levodopa-carbidopa treatment.

\section{CASE REPORT}

A 79-year-old right-handed man was seen for a 5-year history of resting lefthand tremor, jaw tremor and a bradykinetic gait in addition to a remote history of REM-sleep behavior disorder. There was no history of neuroleptic use, or rheumatologic disease.

On examination, he demonstrated hypomimia, hypophonia, a 4- $\mathrm{Hz}$ jaw tremor, resting left pill-rolling tremor, cog-wheeling rigidity in the left arm, and bradykinesia with left arm and leg movements. He had a stooped, shuffling gait with diminished arm swing, and turned en bloc. His neurologic examination was otherwise unremarkable. Laboratory studies (including complete blood count, comprehensive metabolic panel, B12, and serologic testing for syphilis and human immunodeficiency virus) were unremarkable. Brain MRI was declined due to severe claustrophobia.

He was diagnosed with idiopathic Parkinson disease, and started on oral carbidopa/levodopa (Sinemet ${ }^{\circledR}$ ) $25 \mathrm{mg} / 100 \mathrm{mg}$ three times daily. After approximately one week of taking carbidopa/levodopa as prescribed, he experienced severe right shoulder pain, as well as bilateral knee pain and swelling, which were severe enough to cause significant limitation of movement, compelling him to stop taking the medication. The pain and swelling subsequently resolved over the course of a few days upon discontinuation of levodopa/carbidopa.

Approximately three weeks later, he was re-assessed in the neurology clinic. It was felt that the joint pain/swelling was coincidental, and he agreed to re-initiate levodopa/carbidopa therapy. However, three days after restarting the medication, he experienced right shoulder, elbow, wrist and knee pain and swelling. His wife attested to the swelling of these joints. In addition, he reported rib cage pain with inspiration, as well as arthralgias involving his left elbow, wrist, and knee. Again, the pain was severe enough to interfere with mobility and activities of daily living. Upon cessation of levodopa/carbidopa, the pain and swelling subsided over the course of four days.
When he was seen in clinic a week later, moderate right knee pain and crepitus on movement was noted, but he had no obvious joint swelling, redness, or warmth. Alternate treatment with ropinirole or pramipexole was offered, but he declined this.

\section{DISCUSSION}

Using the probability scale described by Naranjo et al for estimating the probability of adverse drug reactions [1], the score for this case is 8 (definite $\geq 9$; probable 5-8; possible 1-4; doubtful $\leq 0$ ), indicating that polyarthralgia in this patient is a probable adverse reaction to levodopa/carbidopa.

Shoulder pain is a common complaint in PD patients. In a study of $25 \mathrm{pa}-$ tients with PD, $80 \%$ experienced either unilateral or bilateral aching shoulder pain that was not typically related to prior shoulder injury (unlike controls). Following treatment with levodopa or dopamine agonists, $40 \%$ reported improvement of the pain whereas the remainder reported no or uncertain effect. None had reported worsening of the pain [2].

In randomized, controlled clinical trials, shoulder pain was reported in $1 \%$ of patients on sustained-release carbidopa/levodopa $(n=491)$ compared with $0.6 \%$ of those on immediate-release carbidopa/levodopa $(n=524)[3,4]$. To date, no clear pathophysiologic mechanism has been proposed for this shoulder pain. DrugInformer (a website that began as a joint project between the Information Science Department and School of Pharmacology at the University of Utah, with subsequent involvement of Aculus LLC) lists 128 reports of arthralgias as an adverse effect of carbidopa-levodopa, without specific details of the cases [5].

Arthralgia in this patient was not limited to the shoulder but involved multiple joints. One plausible explanation for arthralgia in this patient may be a reaction to the deposition of dihydroxyphenylalanine (DOPA) in the joints. Selective darkening of costal cartilage was first reported in a post-mortem examination of a 70-year-old woman with PD on prolonged levodopa/carbidopa therapy (13 years). Histologically, diffuse deposition of black pigment throughout the matrix of the cartilage, and the presence of DOPA was confirmed by high-performance liquid chromatography [6]. Of particular interest, selective darkening of the costal cartilage was also observed in a series of 12 autopsy cases who had been on chronic levodopa therapy (at least 6 years); most used benserazide hydrochloride, a DOPA decarboxylase inhibitor [7]. While irreversible, this discoloration of cartilage with levodopa therapy appears to be harmless, and without any clinical correlations $[6,7]$. There are several limita- 
tions with this hypothesis. Firstly, his joint pain began a few days after starting levodopa whereas blackened cartilage was observed in prolonged therapy. Secondly, while our patient's complaints involved multiple joints, selective costal cartilage involvement was seen in these cases. Thirdly, the blackening of costal cartilage in these cases were asymptomatic.

The second possible explanation is levodopa-induced arthritis/arthralgia, via aggravation of a pre-existing arthritic condition, and precipitation of a transient syndrome in previously healthy joints [8]. This patient had no history of autoimmune rheumatologic disease, but he probably had undiagnosed osteoarthritis affecting some joints. It is possible that a reaction to levodopa in these previously-damaged joints resulted in polyarthralgia/arthritis. However, this would not account for the involvement of joints that are typically not affected by osteoarthritis (e.g., costochondral joints); such joints may have been directly affected by a transient articular or systemic syndrome provoked by levodopa/carbidopa.

\section{LIMITATIONS}

The possibility that the patient's joint pains represent an adverse reaction to inactive ingredients (excipients) in Sinemet ${ }^{\circledR}$ cannot be completely ruled out. He was taking metoprolol 100 mg twice daily for hypertension; while there are no reported drug-drug interactions with levodopa-carbidopa, an unusual interaction causing polyarthralgias cannot be completely ruled out.

\section{CONCLUSION}

In summary, this case describes polyarthralgia as a probable adverse reaction to levodopa-carbidopa therapy. It is important for neurologists to be aware of this potential reaction and consider alternatives to this medication (e.g., dopamine agonists) in those patients developing polyarthralgias with levodopa-carbidopa.

\section{ARTICLE INFORMATION}

*Correspondence: Shin C. Beh, MD, Department of Neurology, University of Texas, Southwestern Medical Center, 5323 Harry Hines Blvd, Dallas, TX 75390-9036, USA. E-mail: shin.beh@utsouthwestern.edu
Received: Jul. 15, 2018; Accepted:Jul. 30, 2018; Published: Mar. 04, 2019

DOI: 10.24983 / scitemed.nnr.2019.00104

Acknowledgement: The author would like to acknowledge Dr. Olaf Stuve's advice in preparing this manuscript.

Disclosures: No relevant financial disclosures.

Ethics Approval and Consent to Participate: The study is in accordance with the ethical standards of the 1964 Helsinki declaration and its later amendments or comparable ethical standards.

Funding: The drafting of this manuscript received no financial support from industry or the National Institutes of Health.

Conflict of Interest: The authors report no financial or other conflict of interest relevant to this article, which is the intellectual property of the authors.

Copyright (c) 2019 The Author (s). This is an open-access article distributed under the terms of the Creative Commons Attribution 4.0 International License (CC-BY).

\section{REFERENCES}

1. Naranjo CA, Busto $U$, Sellers EM, et al. A method for estimating the probability of adverse drug reactions. Clin Pharmacol Ther 1981;30(2):239-245.

2. Madden MB, Hall DA. Shoulder pain in parkinson's disease: A case-control study. Mov Disord 2010;25(8):1105-1106.

3. Sinemet ${ }^{\circledR}$ CR (carbidopa-levodopa) [package insert] Morgantown, WV: Mylan Pharmaceuticals, Inc; 2014. Available at: https://nww.accessdata.fda.gov/drugsatfda_docs/label/2014/019856s016s024s028lbl.pdf. Accessed March 03, 2019.

4. Sinemet ${ }^{\circledR}$ (carbidopa-levodopa) prescribing information. Whitehouse Station, NJ: Merck \& Co., Inc. Available at: https://uww.accessdata.fda.gov/drugsatfda_docs/ label/2008/017555s069|bl.pdf. Accessed March 03, 2019.

5. Druginformer.com. Carbidopa levodopa related arthralgia. Available at: https://druginformer.com/search/side_effect_details/duopa/arthralgia.html. Accessed March 03, 2019.

6. Connolly CE, O'Reilly U, Donlon J. Black cartilage associated with levodopa. Lancet 1986;1(8482):690.

7. Rausing A, Rosen U. Black cartilage after therapy with levodopa and methyldopa. Arch Pathol Lab Med 1994;118(5):531-535.

8. Hart FD. Drug-induced arthritis and arthralgia. Drugs 1984;28(4):347-354. 